\title{
Device-Independent Relativistic Quantum Bit Commitment
}

\author{
Emily Adlam ${ }^{1}$ and Adrian Kent ${ }^{1,2}$ \\ ${ }^{1}$ Centre for Quantum Information and Foundations, DAMTP, Centre for Mathematical Sciences, \\ University of Cambridge, Wilberforce Road, Cambridge, CB3 0WA, U.K. \\ ${ }^{2}$ Perimeter Institute for Theoretical Physics, 31 Caroline Street North, Waterloo, ON N2L 2Y5, Canada.
}

(Dated: April 2015)

\begin{abstract}
We examine the possibility of device-independent relativistic quantum bit commitment. We note the potential threat of location attacks, in which the behaviour of untrusted devices used in relativistic quantum cryptography depends on their space-time location. We describe relativistic quantum bit commitment schemes that are immune to these attacks, and show that these schemes offer device-independent security against hypothetical post-quantum adversaries subject only to the no-signalling principle. We compare a relativistic classical bit commitment scheme with similar features, and note some possible advantages of the quantum schemes.
\end{abstract}

\section{Introduction}

This paper explores the scope for device-independent relativistic quantum cryptographic protocols, focussing on the case of relativistic quantum bit commitment. There have been considerable advances lately in both relativistic quantum cryptography [8 28] and device-independent quantum cryptography [29 46]. This motivates our investigation of device-independent relativistic protocols. Such protocols are not only potentially practically useful but also theoretically interesting, because they add to our understanding of the relationship between quantum nonlocality, cryptographic security and the no-signalling principle. For example, asking whether certain correlations are "sufficiently nonlocal" to implement a given task securely suggests useful measures of nonlocality. Determining which tasks can be implemented using quantum or no-signalling resources is also a path to understanding the properties of relativistic quantum information [11].

For tasks such as bit commitment, which involve two mistrustful parties, device-independent protocols aim to guarantee security to both parties even when neither of them trusts the quantum devices used. Fully device-independent protocols make no assumption whatsoever about the mechanisms of the devices used, except that (a) they are constrained by known physical laws, (b) they can be contained within secure laboratories, whose resident cryptographer controls all inward and outward information flows. Thus both parties must allow for the possibility that any quantum devices they use may have been designed by the other party, who may maliciously have included hidden features designed to give the appearance of normal functioning while actually compromising security.

One point we think particularly worth highlighting is that requiring full device-independence is an even stronger constraint in relativistic quantum cryptography than in standard quantum cryptography. Relativistic cryptographic protocols require specific configurations of agents and devices in space-time, arranged to ensure that the agents cannot exchange data during the protocol (or critical parts thereof). Thus if two separated agents are using devices that are supposed to produce appropriately correlated outputs, they may be unable to check this behaviour during critical parts of the protocol. Even if they perform tests of the devices before the protocol, they remain vulnerable to space-time location attacks. That is, devices that are able to track their own space and time coordinates may be programmed to reproduce the expected behaviour when they are originally tested but not when they are implementing a critical part of the protocol at specified space-time locations. For example, if a particularly critical bit commitment is going to be initiated at noon GMT by devices in Cambridge and Auckland, one of Alice's devices might be programmed so that it usually behaves as Alice expects but, if and only if it is in Cambridge at noon, it gives her an output that should encrypt her committed bit but actually immediately reveals it to Bob's Cambridge agent. These attacks are potentially problematic, because they defeat some testing strategies that work well in non-relativistic device-independent quantum cryptography. Nonetheless, the protocols we describe resist them.

From the practical point of view, one would also like to understand whether device-independent relativistic quantum bit commitment protocols have any advantage over relativistic classical bit commitment protocols. Device-independent protocols aim to ensure security despite a lack of trust in quantum devices, but still require trusted classical computing, storage and communication devices. Classical bit commitment protocols also require trusted classical devices, but require no quantum devices. It is well known that there are unconditionally secure classical relativistic bit commitment protocols [8, 9]. While these involve somewhat different configurations of agents in space-time from the deviceindependent protocols we consider here, we describe below another unconditionally secure classical relativistic bit commitment protocol that has the same configuration of agents as our device-independent protocols. Our deviceindependent quantum schemes thus cannot offer any advantage on this point. However, relativistic quantum bit commitment protocols do have some subtler advantages in some scenarios [25, 28], which one can seek to maintain in device-independent versions. We discuss these further below.

Device-Independent Relativistic Quantum Bit Commitment Protocols 
We begin by considering device-independent versions of the two entanglement-based relativistic bit commitment protocols described in Ref. [28]. The protocols of Ref. [28] are unconditionally secure given the validity of quantum mechanics and special relativity, assuming that the participating agents' trust the functionality of their quantum preparation and measuring devices, up to limited losses and errors. Here we describe variations whose security does not require trusted devices. These protocols can be proven secure assuming only the cryptographic no-signalling principle [30]:

Definition 1. No-signalling principle: Let $D_{i}$ (for $1 \leq i \leq N$ ) be devices in separate secure laboratories at points $P_{i}$, which accept inputs $A_{i}$ and produce outputs $X_{i}$. Let $J$ be any subset of $\{1 \ldots N\}$, let $\bar{J}$ be the complementary subset, and let $X_{J}$ be the random variable $\left\{X_{j}\right\}_{j \in J}$. Then the conditional probability distributions for the outcomes satisfy $p\left(X_{J} \mid A_{1}, \ldots, A_{N}\right)=p\left(X_{J} \mid A_{J}\right){ }^{1}$

Since our security proofs make no assumptions about the properties of preparation or measurement devices, these protocols offer device-independent security. Moreoever, the proofs do not assume the validity of quantum mechanics, and therefore these protocols are also secure against adversaries who can exploit hypothetical post-quantum nonsignalling theories.

Bit commitment We employ the definitions set out in Ref. [28] for bit commitment and relativistic bit commitment, and refer to the discussion therein for further commentary.

In the protocols we consider below, there are two unveiling agents $A_{0}$ and $A_{1}$, whose actions are spacelike separated from each other and from those of $A_{c}$. The probability of a successful unveiling of bit value $i$ depends only on the actions of agent $A_{i}$. We will say that such a protocol provides unconditional, device-independent security against Alice if any only if for any collective strategy $S$ which is possible according to the no-signalling principle, $p_{0}(S)+p_{1}(S)<1+\epsilon(N)$ and $\epsilon(N) \rightarrow 0$ as $N \rightarrow \infty$, where $N$ is a variable security parameter of the protocol, and $p_{0}(S)$ and $p_{1}(S)$ are the probabilities that, by following strategy $S$, Alice and her agents persuade Bob that they have validly committed and unveiled 0 or 1 respectively according to the rules of the protocol. Note that a collective strategy may be fixed by Alice before the protocol, or Alice's agents responsible for unveiling 0 and 1 may independently choose their strategies after the commitment time, possibly conditioned on events in the past lightcone of their verification point but not of the commitment point. We subsume the latter possibility under the former by allowing strategy $S$ to include steps in which agents make strategic choices with probabilities conditional on certain external events, with those events themselves now explicitly included in the description of strategy $S$. Any strategy whereupon the conditional probabilities for these choices are nontrivial may be written as a convex combination of deterministic strategies, so no probabilistic strategy can have greater success probability than the most successful deterministic strategy.

We say that a relativistic protocol is unconditionally secure against Bob if, for any strategy Bob's agents may follow that is consistent with the no-signalling principle, if Alice's agents choose not to unveil, then the probability of any of Bob's agents correctly guessing the committed bit at any point in space-time is bounded by $1 / 2+\epsilon^{\prime}(N)$, where $\epsilon^{\prime}(N) \rightarrow 0$ as $N \rightarrow \infty$. It follows from this definition, by the no-signalling principle, that when Alice does choose to unveil, Bob cannot guess Alices commitment anywhere that does not lie in the future lightcone of the unveiling points.

In the protocols we consider below, Alice has one committing agent, $A_{c}$, and two unveiling agents, $A_{0}$ and $A_{1}$, who can unveil a valid commitment to $b=0$ and 1 respectively. An additional security criterion may be required for such protocols: that if $A_{c}$ does not make a valid commitment to bit value $b, A_{b}$ follows the unveiling protocol and $A_{\bar{b}}$ does not, then Bob's agents, at any point in space-time, should gain no information about whether $A_{c}$ committed to bit value $\bar{b}$ or declined to make a valid commitment. Our protocols also satisfy this criterion.

As usual in quantum cryptography, we initially present our protocols in an idealized form assuming perfect quantum state preparations, transmissions, measurements and computations. However, the protocols are tolerant to noise and errors, as we discuss later.

Space-time and communications We also make standard idealizations about the background geometry and signalling speed. We suppose that space-time is Minkowski and that Alice and Bob each have agents in secure laboratories infinitesimally separated from the points $P, Q_{0}$ and $Q_{1}$, that signals are sent at precisely light speed, and all information processing is instantaneous. Again, these assumptions can be relaxed. The protocols remain secure in realistic implementations with finite separations and near light speed communication. If these corrections are small, the only significant effect is that Bob is guaranteed that Alice's commitment is binding from some point $P^{\prime}$ in the near causal future of $P$, rather than from $P$ itself [13]. Allowing for small deviations from Minkowski geometry

\footnotetext{
${ }^{1}$ If both inputs and outputs take place at the same points $P_{i}$, then ensuring that these points are spacelike separated is a sufficient condition. It is not necessary, however. The cryptographic assumption of secure laboratories implies that the devices cannot communicate input or output information in any way through the laboratory walls, whether or not they are spacelike separated.
} 
also requires small corrections to the geometry when stating the security guarantees, but does not essentially affect security beyond that $[9]$.

Geometry Alice and Bob agree on a space-time point $P$ and an inertial set of coordinates $(x, y, z, t)$ for Minkowski space with $P$ as the origin. We focus here on the simplest case in which there are two possible unveiling points $Q_{0}$ and $Q_{1}$; the protocols straightforwardly extend to versions with $N$ unveiling points committing $\log (N)$ bits. Alice and Bob each have agents, who during the protocol are separated in secure laboratories, adjacent to each of the points $P, Q_{0}, Q_{1}$. To simplify we take the distances from these labs to the relevant points as negligible. Let the agents adjacent to $P$ be $A_{c}$ and $B_{c}$, and those adjacent to $Q_{i}$ be $A_{i}$ and $B_{i}$.

For the two protocols we describe here, the unveiling points must be spacelike separated from the commitment point. Although it is not necessary for much of our discussion, we assume that $Q_{0}$ and $Q_{1}$ have positive time coordinates in the given frame, and therefore these protocols are fixed frame positive duration (FFPD) relativistic bit commitments in the terminology of Ref. [28].

We will also suppose that Bob has an unlimited number of additional agents which he may position at various points between the the commitment point $P$ and the unveiling points $Q_{0}$ and $Q_{1}$. However Bob needs these agents only if he is concerned about verifying Alice's commitment as soon as possible; if the situation is not time-sensitive the verification steps described below could be carried out either by $B_{c}$ or $B_{i}$ at a slightly later time.

For definiteness we describe a protocol in which Alice and her agents exchange qubits by physical transportation in the preparation phase. They may alternatively employ a secure quantum channel without materially altering the security of the protocol. Note however that assuming a physically secure quantum channel requires that the physical path of the channel lies within Alice's secure laboratories. If one instead supposes they use quantum teleportation, then to make a fair comparison of a fully device-independent scheme against alternatives, one would need to discuss further the trust Alice places in her quantum teleportation devices and the encryption or other resources she may consume.

Notation For the security proofs we employ a binary notation, denoting measurement directions $X, Y$ by 0,1 , measurement directions $X^{\prime}, Y^{\prime}$ also by 0,1 , and measurement outcomes $+1,-1$ also by 0,1 . A single Bell experiment is then described by a choice of two measurement settings $(x, y) \in\{0,1\}$ and two measurement results $(O, s) \in\{0,1\}$. We are interested in the CHSH game score $R(t \oplus s=x y)$, which is the number of experiments in the set for which the settings $(x, y)$ and outcomes $(t, s)$ satisfy the condition $t \oplus s=x y$. In the case when the settings are random and independent, the CHSH game score is asymptotically related to the average value of the CHSH observable $\left(X X^{\prime}+X Y^{\prime}+Y X^{\prime}-Y Y^{\prime}\right)$ : for a set of $N$ experiments, the latter expression is $\frac{4}{N}(2 R(t \oplus s=x y)-N)$ to leading order in $N$. However, we do not assume independent random settings in all of our protocols.

CHSH 1: CHSH test protocol with fixed directions

Preparation 1. Alice and Bob agree on a set of four directions $X, Y, X^{\prime}, Y^{\prime}$ such that all four directions lie in a single plane, $X$ is orthogonal to $Y, X^{\prime}$ is orthogonal to $Y^{\prime}, X^{\prime}$ is separated from $X$ by $\frac{\pi}{4}$ and $Y^{\prime}$ is separated from $Y$ by $\frac{\pi}{4}$. They also agree on a bit string $L^{0}$ with bitwise complements $L^{1}$.

2. $A_{c}$ instructs her devices to prepare $2 N$ Bell pairs $\left(W_{a}^{i}, W_{b}^{i}\right)$ in the singlet state $\Psi^{-}$, then randomly draws an integer $x \in\{0,1\}$. If $x=0$ she gives the particles $\left[W_{b}^{1}, \ldots W_{b}^{N}\right]$ to an agent $A_{0}$ and the remaining particles $\left[W_{b}^{N+1}, \ldots W_{b}^{2 N}\right]$ to an agent $A_{1}$; if $x=1$ she instead gives the particles $\left[W_{b}^{N+1}, \ldots, W_{b}^{2 N}\right]$ to $A_{0}$ and the particles $\left[W_{b}^{1}, \ldots, W_{b}^{N}\right]$ to $A_{1} . A_{0}$ and $A_{1}$ then travel to points $Q_{0}$ and $Q_{1}$ both at a distance $d$ away from $A_{c}$.

We assume that $A_{c}, A_{0}$ and $A_{1}$ have secure laboratories that protect their qubits, so $B_{c}$ cannot interfere with them in any way after the initial preparation. In particular, $A_{0}$ and $A_{1}$ travel within secure laboratories. We also assume that the first and second sets of states (i.e. the purported half-singlet states labelled $a$ and $b$ respectively) are securely separated in sub-laboratories before $A_{c}$ chooses the value of $x$. Thus, even if the $a$ states are actually malicious devices operating within the laboratory, they obtain no information about the value of $x$ or the destinations of the $b$ states.

Commitment At the designated commitment point $P, B_{c}$ gives $A_{c}$ a bit string $L$ drawn uniformly at random from $\mathbb{Z}_{2}^{\otimes N}$. If $A_{c}$ wants to commit to 0 , she instructs her devices to measure each qubit $W_{a}^{j+x N} \in\left[W_{a}^{1+x N}, \ldots, W_{a}^{N+x N}\right]$ in direction $(X)^{L_{j}}(Y)^{1-L_{j}}$. If $A_{c}$ wants to commit to 1 she instead instructs her devices to measure each qubit $W^{N+j-x N} \in\left[W_{a}^{N+1-x N}, \ldots, W_{a}^{2 N-x N}\right]$ in direction $(X)^{L_{j}}(Y)^{1-L_{j}}$. In either case $A_{c}$ immediately broadcasts her outcomes.

Unveiling For $i \in\{0,1\}$ :

At the point $Q_{i}$, if agent $A_{i}$ believes Alice wishes to unveil, she measures each qubit $W_{a}^{j+x N+i N-2 i x N} \in$ $\left[W_{a}^{1+x N+i N-2 i x N} \ldots W_{a}^{N+x N+i N-2 i x N}\right]$ in direction $\left(X^{\prime}\right)^{L_{j}^{i}}\left(Y^{\prime}\right)^{1-L_{j}^{i}}$. She then broadcasts her measurement outcomes.

Note that in principle the agents $A_{c}, A_{0}$ and $A_{1}$ may make these decisions independently. If Alice wishes to coordinate them and ensure that all or none unveil, she needs to give them instructions in advance. These instructions could depend on separate events in the past light cones of their unveiling decision points, if Alice believes these events will be correlated.

Verification Bob's agents between $P$ and $Q_{i}$ wait for the data from $A_{c}$ and $A_{i}$. As soon as one agent receives both sets of data, he calculates the CHSH game score. If the result is greater than $N((2+\sqrt{2}) / 4-\xi)$ he accepts that Alice 
made a valid commitment to bit value $i$. Here $\xi$ is some predetermined small security parameter, chosen such that $\xi \gg N^{-1 / 2}$; this ensures that the probability that $N$ correctly prepared and measured singlets will fail Bob's test is suitably small.

Security against Alice $A_{0}, A_{c}$ and $A_{1}$ announce their outcomes at spacelike separation from one another during the protocol. They may pre-agree a collective strategy $S$, which may rely on shared quantum or (hypothetical) postquantum no-signalling resources, but cannot signal to one another during the protocol. We are interested in bounding the probability that $A_{0}$ and $A_{1}$ both succeed in passing Bob's tests for a valid commitment of 0 and 1 respectively.

Let $p_{0}(S)$ and $p_{1}(S)$ be the probabilities that when Alice employs strategy $S$, she and her agents convince Bob that they have have unveiled 0 or 1 respectively according to the rules of the protocol above. Note that these rules allow for the possibility that Bob accepts valid commitments to both 0 and 1 if both tests are passed. Let $p_{0}(S)+p_{1}(S)=1+\epsilon_{S}^{1}(N)$.

Hence for any strategy $S, \epsilon_{S}^{1}(N) \leq \sum_{L} \frac{p_{L, L_{0}}^{S}}{2^{N}}$ where $p_{L, L_{0}}^{S}$ is the probability that in this protocol (defined by $L_{0}$ ), when Alice employs strategy $S$ and Bob chooses the bit string $L$, Alice and her agents produce three sets of outcomes $O, O^{0}$ and $O^{1}$ such that:

$$
\begin{aligned}
& d\left(O^{0} \oplus O, L^{0} L\right) \leq N\left(\frac{1}{2}-\frac{1}{2 \sqrt{2}}+\xi\right) \\
& d\left(O^{1} \oplus O, L^{1} L\right) \leq N\left(\frac{1}{2}-\frac{1}{2 \sqrt{2}}+\xi\right)
\end{aligned}
$$

Here $d(x, y)$ denotes the Hamming distance between the bit strings $x$ and $y$, which is the number of positions on which the strings differ, $O^{i} \oplus O$ is the string given by element-wise modular addition of $O^{i}$ and $O$, and $L^{i} L$ is the string given by element-wise multiplication of $L^{i}$ and $L$.

These equations can be simultaneously satisfied only if $d\left(O^{0} \oplus O^{1}, L\left(L^{0} \oplus L^{1}\right)\right) \leq N\left(1-\frac{1}{\sqrt{2}}+2 \xi\right)$. Since $L^{0}$ is the bitwise complement of $L^{1}$, this implies that $d\left(O^{0} \oplus O^{1}, L\right) \leq N\left(1-\frac{1}{\sqrt{2}}+2 \xi\right)$.

Thus for given $O^{0} \oplus O^{1}$, equations 1 and 2 may be satisfied simultaneously only if $L$ lies within the Hamming ball $H$ of radius $r=N\left(1-\frac{1}{\sqrt{2}}+2 \xi\right)$ centered on $O^{0} \oplus O^{1}$. For $r \leq \frac{N}{2}$, the volume of this ball is less than or equal to $2^{N H(r / N)}$ where $H(x)=-x \log (x)-(1-x) \log (1-x)[3]$.

$H(x) \leq 1$ with equality if and only if $x=\frac{1}{2}$, so for any $\xi<\frac{1}{2 \sqrt{2}}-\frac{1}{4}$ we have $H(r / N)<1$.

By the no-signalling principle, the probability distribution for $O^{0} \oplus O^{1}$ is independent of $L$. Thus since $L$ is chosen uniformly at random from $\mathbb{Z}_{2}^{\otimes N}$, we must have $\epsilon_{S}^{1}(N) \leq 2^{-N(1-H(r / N))}$, which for $H(r / N)<1$ goes to zero as $\mathrm{N}$ goes to infinity.

\section{Security against Bob}

Alice's devices may perhaps have been designed by Bob in an attempt to cheat the protocol. If the devices do something other than performing $\mathrm{CHSH}$ measurements on a shared quantum singlet, $A_{c}$ 's outputs may give $B_{c}$ information about whether $A_{c}$ measured the qubits $\left[W_{a}^{1} \ldots W_{a}^{N}\right]$ or $\left[W_{a}^{N+1} \ldots W_{a}^{2 N}\right]$. This may allow $B_{c}$ to update his prior values (which are originally equiprobable) for $P(b \mid x)$, the conditional probability of the committed bit value $b$ given the value of $A_{c}$ 's randomly chosen bit $x$. However, by assumption, $x$ is random and kept secret throughout the protocol. Before receiving unveiling data, Bob's estimate of $P(b)=\frac{1}{2}(P(b \mid 0)+P(b \mid 1))$ thus remains unaltered.

Security against Bob thus relies on Alice being able to generate one unconditionally secure random bit per committed bit, and to store this bit securely in $A_{c}$ 's laboratory during the protocol.

CHSH2: CHSH protocol with secret complementary bit strings CHSH1 may straightforwardly be varied so that Bob keeps the bit strings $L^{0}$ and $L^{1}$ secret from Alice until the points $Q^{0}$ and $Q^{1}$ :

Preparation 1. $B_{c}$ chooses a length $N$ bit string $L^{0}$ drawn uniformly at randomly from $\mathbb{Z}_{2}^{\otimes N}$. He communicates string $L^{0}$ to $B_{0}$ and its bitwise complement $L^{1}$ to $B_{1}$.

The remaining steps are identical to those for CHSH1.

Security against Alice: The only change in this protocol is that Alice is given less information, which can only decrease her probability of cheating successfully. Hence the security of this protocol against Alice follows immediately from the security of CHSH1 against Alice.

Security against Bob The proof of security against Bob is the same as for CHSH1.

\section{CHSH3: CHSH test protocol with full randomisation}


CHSH1 may also be varied so that the measurement directions for $A_{0}$ and $A_{1}$ are not fixed in advance. Instead, each $B_{i}$ randomly selects a set of $N$ measurement directions to be used by $A_{i}$, so the string $L^{1}$ is no longer guaranteed to be the bitwise complement of $L^{0}$.

The steps in this protocol are identical to those for CHSH1 except for the unveiling stage:

Unveiling For $i \in\{0,1\}$ :

At the point $Q_{i}, B_{i}$ gives $A_{i}$ a bit string $L^{i}$ drawn uniformly at randomly from $\mathbb{Z}_{2}^{\otimes N}$. If agent $A_{i}$ believes Alice wishes to unveil, she immediately measures each qubit $W_{a}^{j+x N+i N-2 i x N} \in\left[W_{a}^{1+x N+i N-2 i x N} \ldots W_{a}^{N+x N+i N-2 i x N}\right]$ in direction $\left(X^{\prime}\right)^{L_{j}^{i}}\left(Y^{\prime}\right)^{1-L_{j}^{i}}$. She then broadcasts her measurement outcomes.

Security against Alice

As before, for any strategy $S$, let $p_{0}(S)$ and $p_{1}(S)$ be the probabilities that when Alice employs the strategy, Alice's agents convince Bob that they have have validly unveiled 0 or 1 respectively according to the rules of the protocol, and suppose $p_{0}(S)+p_{1}(S)=1+\epsilon_{S}^{2}(N)$.

By the no-signalling principle, $A_{0}$ 's success probability is independent of the choice of $L_{1}$, and $A_{1}$ 's success probability is independent of the choice of $L_{0}$. Hence we may calculate $p_{0}(S)$ and $p_{1}(S)$ assuming that $L_{0}$ and $L_{1}$ are bitwise complements. We thus obtain the same bounds on $p_{0}(S)+p_{1}(S)$ as for the first two protocols.

Security against Bob The proof of security against Bob is the same as for CHSH1.

\section{Extensions}

\section{Declining to commit}

In these relativistic bit commitment schemes, $A_{c}$ decides whether or not to make a commitment. If she makes a decision at the point $P$, she cannot communicate it to the $A_{i}$ before they make decisions about whether or not to unveil. Bob will learn whether or not a commitment was made and validly unveiled if he receives unveiling data. However, Alice may perhaps not wish Bob to learn whether or not $A_{c}$ chose to make a valid commitment in the cases when the $A_{i}$ choose not to unveil.

A subtle potential drawback of the protocols described above is that the security proofs do not guarantee that Bob will be unable to tell whether or not $A_{c}$ has chosen to make a commitment. If Alice's devices actually implement CHSH measurements on singlets, then $A_{c}$ could decline to commit simply by returning a random string of $N$ bits to $B_{c}$. However, Bob may have designed Alice's preparation devices. He thus may be able to distinguish between randomly chosen bit strings and strings produced by measurements on Alice's Bell pairs, and may therefore may be able to detect if $A_{c}$ tries this tactic to refrain from making a commitment.

However, in scenarios where these feature is problematic, our protocols can straightforwardly be altered to eliminate it. Alice and Bob may simultaneously perform two runs of $\mathrm{CHSH} 1$, with the rule that Alice commits to bit value $b$ in both protocols if she wishes to produce a valid commitment to $b$, but commits to different values in each protocol if she wishes to refrain from commitment. Bob will then accept that Alice has made a valid commitment to bit value $b$ only if she unveils valid commitments to bit value $b$ for both protocols. CHSH2 or CHSH3 may similarly be duplicated in this way.

Bob obtains no information about the values of $x$ used in the two protocols unless Alice unveils. He thus cannot tell whether or not she made a valid commitment if she declines to unveil.

\section{Errors and losses}

We now consider how to allow for the possibility of small errors in preparations, transmissions and measurements of quantum states. We consider an error model which allows for errors and losses in the preparation, transmission and measurement of singlet states. We suppose that any such errors occur randomly and independently, and that the combined rate of errors and losses is bounded by a parameter $\delta$, which we assume is small. In this model, the expectation values of the distances $d\left(O^{i} \oplus O, L^{i} L\right)$ are altered by no more than $\delta N$, with variance no larger than $\delta^{1 / 2} N^{1 / 2}$.

Bob's verification tests involve a security parameter $\xi$, and the security proof for CHSH1 holds for

$$
\xi<\frac{1}{2 \sqrt{2}}-\frac{1}{4} .
$$

In particular, if $\delta$ is agreed to be the maximum tolerable rate of errors and losses, and $\delta$ is small, Bob can use a value $\xi>\delta$ in his verification tests, consistent with (3), and still ensure security against Alice.

The security proofs of CHSH2 and CHSH3 do not depend on additional assumptions about errors and losses, and so similarly remain secure for small $\delta$. Thus we can ensure that both protocols are secure against Alice provided the expected rate of errors and losses is small.

The proofs of security against Bob do not depend on any assumptions about errors or losses, and therefore no alteration is required for these proofs. 


\section{Discussion}

\section{Device memory attacks}

A quite general concern in device-independent quantum cryptography is that maliciously designed devices may keep records of their inputs and outputs and may make their future outputs depend on these data [40]. Our protocols are secure against these attacks for a single bit commitment. However, if Alice reuses her devices for a sequence of commitments, our protocols do not protect her from the possibility that data released (for example to $B_{c}$ ) in a later commitment may give information about earlier commitments. This is not a concern if all her commitments are in any case later unveiled, so long as the devices are not reused until points in the causal future of points at which Bob can receive both the commitment and unveiling data. Similarly, it would not be a concern if her commitment data loses any value in the causal future of certain spacetime points (call them expiration points), whether or not it was unveiled, so long as the devices are not reused until points in the causal future of an expiration point. However, in many common scenarios, some commitment data is unveiled and some should be kept secret indefinitely. Our protocols do not guarantee security for device reuse against completely general attacks in such scenarios.

However, our protocols do guarantee security for device reuse against memory attacks, so long as the devices are not also able to carry out location attacks, and so long as the $A_{i}$ 's decision whether to unveil is coordinated. The reason is that, if neither $A_{0}$ and $A_{1}$ accept any device input or supply any output unless the bit is to be unveiled, and if the devices are not sensitive to their location, then the devices can carry no information correlated with $x$ (and hence nor with $b$ ) after a protocol without unveiling. They thus have no ability to leak information about $b$ in future protocols.

Other security assumptions

As with the protocols of Refs. [9, 13, 22], the present protocols can be chained together in sequence, allowing longer term bit commitments and flexibility in the relation between the commitment and unveiling sites (in particular, they need not be lightlike separated). Full security and efficiency analyses for these chained protocols remain tasks for future work.

We note that the unconditional, device-independent security of both protocols depend on the assumption that Bob has access to a device independent method of generating random numbers which is secure in the sense that Alice cannot predict its output in advance. There exists no device independent method of generating random numbers with no random seed, but there do exist device independent methods of randomness expansion [41 43, 45], which can create a long random string from some small random seed. Since the random seed used in these protocols can be of a low quality of randomness (i.e. the expansion still works if the 'random' input is partially correlated with variables which may be known to the adversarial party [47]), it could be obtained by some classical quasi-random process which Alice is unlikely to be able to predict or control perfectly, such as a coin flip. Therefore device-independent randomness expansion suffices for effectively device-independent security of these protocols.

The CHSH2 protocol requires Bob to generate $2 \mathrm{~N}$ random strings and the $\mathrm{CHSH} 3$ protocol requires him to generate $3 N$ random strings, in contrast with only one string in CHSH1. However there may be contexts in which CHSH2 and CHSH3 are preferred - for example, if Bob wishes to control the time of Alice's measurements, which might be particularly important for chained protocols. In the CHSH1 protocol, Alice may make the measurements whose results she unveils at $Q_{0}$ and $Q_{1}$ at any time, even before the start of the protocol, but in CHSH2 and CHSH3 she must wait until Bob tells her which measurement directions to use. In such contexts CHSH3 offers greater security to Bob, because in CHSH2, $L_{1}$ must be the bitwise complement of $L_{0}$ and therefore it follows from the no-signalling principle that $B_{0}$ and $B_{1}$ must agree on these strings at some time in the causal past of $P$. This is a potential security weakness, since if Alice can find out the strings $L_{0}$ and $L_{1}$ in advance of the commitment, then $A_{0}$ and $A_{1}$ can perform their measurements earlier than Bob expects.

Note that, like all technologically unconstrained quantum bit commitment protocols [20, 21], our protocols do not prevent Alice from committing to a quantum superposition of bits. She can simply input a superposition $\alpha|0\rangle+\beta|1\rangle$ into a quantum computer programmed to implement the two relevant quantum measurement interactions for inputs $|0\rangle$ and $|1\rangle$ and to send two copies of the quantum outcome data towards $Q_{0}$ and $Q_{1}$, and keep all the data at the quantum level until (if) she chooses to unveil. This gives her no advantage in stand-alone applications of bit commitment, for example for making a secret prediction: it does, however, mean that one cannot assume that in a task involving bit commitment subprotocols, any unopened bit commitments necessarily had definite classical bit values, even if all unveiled bit commitments produced valid classical unveilings.

In comparing relativistic bit commitment protocols, one needs to consider the configuration of agents in spacetime as well as the resources required. To make a precise comparison, we first describe a classical relativistic bit commitment protocol with the same configuration of agents as the schemes above.

\section{Random code classical bit commitment protocol (RCCBC)}

Preparation 1. Alice and Bob agree on a security parameter $N$; for simplicity we take $N$ to be even.

2. $A_{c}$ generates two independent $N$-bit random classical strings, $S^{0}$ and $S^{1}$, and securely shares string $S^{i}$ with 
agent $A_{i}$.

Commitment At the designated commitment point $P, B_{c}$ gives $A_{c}$ a randomly chosen size $N / 2$ subset $J$ of $\{1, \ldots, N\} . \quad A_{c}$ immediately responds by giving $B_{c}$ the string subset $\left\{S_{j}^{i}: i=0,1 ; j \in J\right\}$. These string elements are sent with their labels $i$ and $j$. To commit to bit value $i, A_{c}$ also sends the complementary string subset $S_{\bar{J}}^{i}=\left\{S_{j}^{i}: j \in \bar{J}\right\}$. These string elements are sent with their labels $j$, but not $i$. Hence $B_{c}$ refers to this string as $S_{\bar{J}}$, with $i$ unknown to him.

Unveiling For $i \in\{0,1\}$ :

At the point $Q_{i}$, if agent $A_{i}$ believes Alice wishes to unveil, she gives $B_{i}$ the complete string $S^{i}$.

Verification On receiving the two substrings $S_{J}^{0}$ and $S_{J}^{1}, B_{c}$ checks that their Hamming distance satisfies $\left|d\left(S_{J}^{0}, S_{J}^{1}\right)-N / 4\right|<C N^{3 / 4}$, where $C>0$ is an agreed parameter independent of $N$. If not, he immediately rejects the commitment. Otherwise, Bob's agents between $P$ and $Q_{i}$ wait for the data from $A_{c}$ and $A_{i}$. As soon as an agent receives data from $B_{i}$ and $B_{c}$, he checks whether the string $S^{i}$ reported by $B_{i}$ is the union of the strings $S_{J}^{i}$ and $S_{\bar{J}}$ reported by $B_{c}$, with matching labels for each element. If it is, he accepts that Alice made a valid commitment to bit value $i$.

Security against Alice $A_{0}, A_{c}$ and $A_{1}$ announce their outcomes at spacelike separation from one another during the protocol. They may pre-agree a collective strategy $S$, which may rely on shared quantum or (hypothetical) postquantum no-signalling resources, but cannot signal to one another during the protocol. We are interested in bounding the probability that $A_{0}$ and $A_{1}$ both succeed in passing Bob's tests for a valid commitment of 0 and 1 respectively.

Let $p_{0}(S)$ and $p_{1}(S)$ be the probabilities that when Alice employs strategy $S$, she and her agents convince Bob that they have have unveiled 0 or 1 respectively according to the rules of the protocol above. Note that these rules allow for the possibility that Bob accepts valid commitments to both 0 and 1 if both tests are passed. Let $p_{0}(S)+p_{1}(S)=1+\epsilon_{S}^{3}(N)$.

Hence for any strategy $S, \epsilon_{S}^{3}(N) \leq \sum_{L} \frac{p_{J}^{S}}{2^{N}}$ where $p_{J}^{S}$ is the probability that when Alice employs strategy $S$ and Bob chooses the subset $J$, Alice's agents produce strings $S^{0}$ and $S^{1}$ such that $\left|d\left(S_{J}^{0}, S_{J}^{1}\right)-N / 4\right|<C N^{3 / 4}$ and also that $S_{j}^{0}=S_{j}^{1}=S_{j}$ for $j \in \bar{J}$.

If Alice's agents succeed in producing strings with these properties, they can infer that the complement $\bar{J}$ of $B_{c}$ 's chosen subset $J$ lies within a subset of size no more than $3 N / 4+C N^{3 / 4}$ of $\{1, \ldots, N\}$. By the no-signalling principle, it follows that $\epsilon_{S}^{3}(N) \rightarrow 0$ as $N \rightarrow \infty$.

Security against Bob

Alice's strings are randomly generated. Bob thus receives no information correlated with the bit value $i$ unless and until at least one of the $A_{i}$ unveils.

Note that this security argument relies on Alice being able to generate $2 N$ unconditionally secure classical random bits per committed bit, to share these bits between $A_{c}$ and the $A_{i}$ before the protocol, and to keep them securely in the agents' laboratories before and during the protocol.

Comparison of Classical and Device-Independent Quantum Relativistic Bit Commitment Protocols

Security The classical and device-independent quantum relativistic bit commitment protocols described above all have the same configuration of agents for the two parties, and are all unconditionally secure. While the quantum protocols do not require either party to trust the quantum devices used, they still require both parties to have trusted classical computing and memory devices. They thus have no advantage over the classical protocol in this respect.

Use of Classical Randomness: Bob: The classical protocols and the CHSH1 quantum protocol both require $B_{c}$ to be able to generate a random string that Alice cannot predict in advance. These strings may be generated at $P$, and immediately handed over to $A_{c}$, so they do not need to be kept secure; nor do they need to be distributed securely to Bob's other agents.

The CHSH2 protocol also requires a second random string to be generated and securely distributed and stored by $B_{0}$ and $B_{1}$. The CHSH3 protocols instead requires $B_{0}$ and $B_{1}$ to generate random independent strings that Alice cannot predict in advance. These strings may be generated at the points $Q_{i}$, and immediately handed over to Alice's agents $A_{i}$, so they do not need to be kept secure; nor do they need to be distributed securely to Bob's other agents.

Use of Classical Randomness: Alice: The classical protocol requires Alice to generate two $N$-bit random strings, to share them securely between $A_{c}$ and $A_{0}$ or $A_{1}$, and to store them securely in the various agents' laboratories. In contrast, the $\mathrm{CHSH}$ protocols each require $A_{c}$ to generate and keep secure a single random classical bit. If the devices are distinguishable, this bit is also shared and stored securely in $A_{0}$ and $A_{1}$ 's laboratories, since the set of physical devices (purported stored qubits) given to these agents depends on the bit value.

In addition, the $\mathrm{CHSH}$ protocols require $A_{c}$ to share devices (purportedly containing sets of qubits) with $A_{0}$ and $A_{1}$, and these devices must also be stored securely in the respective laboratories. If Bob can access all the devices, he can gain information about the committed bit.

This adds nuance to the comparison. If the devices do indeed contain sets of qubits, and each qubit is indeed maximally entangled with a another qubit in another appropriately located device, and the devices do indeed faithfully 
carry out projective CHSH measurements on these qubits, Alice could use them to generate classical random strings shared between $A_{c}$ and each of the $A_{i}$, and these shared strings could be used to implement the classical protocol $R C C B C$. However, since the devices are untrusted, Alice cannot naively implement such a strategy. Moreover, although it may be harder to keep entangled quantum states secure than to keep shared random classical strings secure, the former offers a potentially valuable security advantage. Imagine a scenario in which Alice has a large network of agents who carry out commitments, generally relying on untrusted quantum devices. For example, Alice may be technologically disadvantaged, and so must generally rely on externally supplied devices, which may have been produced by Bob. However, suppose that, at some extra cost, Alice is able, without prior announcement, to introduce trusted quantum devices to verify some of the purported singlets used by her agents. She can then get information about the extent of any potential security breaches. If her agents are using the classical protocol, on the other hand, there is no reliable way for her to verify the privacy of the shared classical random strings she uses. If Bob is able to breach her security, he can gain information about the strings (and hence her bit commitments) without altering them or leaving any other physical trace.

Summary Both our device-independent CHSH quantum relativistic bit commitment protocols and their classical counterpart are unconditionally secure. However, the device-independent protocols appear to have some subtle but real advantages that are potentially valuable in some scenarios. We thus take our discussion as offering an argument for the practical value of device-independent quantum cryptography in relativistic bit commitment, and stronger arguments for its value in more general relativistic cryptographic scenarios. We should, though, underline that we have compared specific device-independent protocols against a specific classical protocol. It remains an open task to identify the optimal protocols of each type (for any of the various natural measures of optimality) and compare them.

\section{Acknowledgments}

This work was partially supported by an FQXi mini-grant and by Perimeter Institute for Theoretical Physics. Research at Perimeter Institute is supported by the Government of Canada through Industry Canada and by the Province of Ontario through the Ministry of Research and Innovation. AK thanks Serge Massar for helpful conversations.

[1] D. Mayers, Unconditionally secure quantum bit commitment is impossible, Phys. Rev. Lett. 78 3414-3417 (1997).

[2] D. Mayers, Unconditionally secure quantum bit commitment is impossible, Proceedings of the Fourth Workshop on Physics and Computation (New England Complex System Inst., Boston, 1996), p. 226.

[3] A. Rudra, Limits to List Decoding Random Codes, Computing and Combinatorics, (Springer Berlin, 2009)

[4] H.-K. Lo and H. Chau, Is quantum bit commitment really possible?, Phys. Rev. Lett. 78 3410-3413 (1997).

[5] H.-K. Lo and H. Chau, Why quantum bit commitment and ideal quantum coin tossing are impossible, Proceedings of the Fourth Workshop on Physics and Computation (New England Complex System Inst., Boston, 1996), p. 76.

[6] D. Mayers, A. Kitaev and J. Preskill, Superselection rules and quantum protocols, Phys. Rev. A 69052326 (2004).

[7] G. D'Ariano, D. Kretschmann, D. Schlingemann, R. Werner, Reexamination of Quantum Bit Commitment: the Possible and the Impossible, Phys. Rev. A 76, 032328 (2007).

[8] A. Kent, Unconditionally secure bit commitment, Phys. Rev. Lett. 83 1447-1450 (1999).

[9] A. Kent, Secure Classical Bit Commitment using Fixed Capacity Communication Channels, J. Cryptology 18 (2005) 313-335.

[10] A. Kent, A No-summoning theorem in Relativistic Quantum Theory, Quantum Information Processing 12 (2) pp 1023-1032 (2013).

[11] A. Kent, Quantum Tasks in Minkowski Space, Class. Quantum Grav. 29 (2012) 224013.

[12] P. Hayden and A. May, Summoning Information in Spacetime, or Where and When Can a Qubit Be? arXiv:1210.0913

[13] A. Kent, Unconditionally Secure Bit Commitment with Flying Qudits, New J. Phys. 13113015 (2011).

[14] A. Kent, Location-Oblivious Data Transfer with Flying Entangled Qudits, Phys. Rev. A 84, 012328 (2011).

[15] R. Colbeck and A. Kent, Variable Bias Coin Tossing, Phys. Rev. A 73, 032320 (2006).

[16] A. Kent, Quantum Tagging for Tags Containing Secret Classical Data, Phys. Rev. A 84, 022335 (2011).

[17] R. Malaney, Phys. Rev. A 81, 042319 (2010).

[18] H. Buhrman et al., arXiv:1009.2490v4 (2011).

[19] A. Kent, W. Munro and T. Spiller, Quantum Tagging: Authenticating Location via Quantum Information and Relativistic Signalling Constraints, Phys. Rev. A 84, 012326 (2011).

[20] A. Kent, Impossibility of unconditionally secure commitment of a certified classical bit, Phys. Rev. A 61 042301 (2000).

[21] A. Kent, Why Classical Certification is Impossible in a Quantum World, Quantum Information Processing, 11 (2), $493-499$ $(2012)$.

[22] A. Kent, Unconditionally Secure Bit Commitment by Transmitting Measurement Outcomes, Phys. Rev. Lett. 109, 130501 (2012). 
[23] S. Croke and A. Kent, Phys. Rev. A 86, 052309 (2012).

[24] J. Kaniewski et al., IEEE Trans. on Inf. Theory 59, 4687-4699 (2013).

[25] T. Lunghi et al., Phys. Rev. Lett. 111, 180504 (2013).

[26] Y. Liu et al., Phys. Rev. Lett. 112, 010504 (2014).

[27] T. Lunghi et al., arXiv:1411.4917

[28] E. Adlam and A. Kent, Deterministic Relativistic Quantum Bit Commitment, arxiv:15mm.nnnnn.

[29] Mayers, D. \& Yao, A. Quantum cryptography with imperfect apparatus. In Proceedings of the 39th Annual Symposium on Foundations of Computer Science (FOCS-98), 503-509 (IEEE Computer Society, Los Alamitos, CA, USA, 1998).

[30] Barrett, J., Hardy, L. \& Kent, A. No signalling and quantum key distribution. Physical Review Letters 95, 010503 (2005).

[31] Acin, A., Gisin, N. \& Masanes, L. From Bell's theorem to secure quantum key distribution. Physical Review Letters 97, 120405 (2006).

[32] Scarani, V. et al. Secrecy extraction from no-signaling correlations. Physical Review A 74, 042339 (2006).

[33] Acin, A. et al. Device-independent security of quantum cryptography against collective attacks. Physical Review Letters 98, 230501 (2007).

[34] Masanes, L., Renner, R., Christandl, M., Winter, A. \& Barrett, J. Unconditional security of key distribution from causality constraints. e-print quant-ph/0606049v4 (2009).

[35] Masanes, L. Universally composable privacy amplification from causality constraints. Physical Review Letters 102, 140501 (2009).

[36] Hänggi, E., Renner, R. \& Wolf, S. Quantum cryptography based solely on Bell's theorem. In Gilbert, H. (ed.) Proceedings of the 29th Annual International Conference on the Theory and Applications of Cryptographic Techniques (Eurocrypt'10), 216-234 (Springer, 2010). Also available arXiv:0911.4171.

[37] Hänggi, E. \& Renner, R. Device-independent quantum key distribution with commuting measurements. e-print arXiv:1009.1833 (2010).

[38] Masanes, L., Pironio, S. \& Acín, A. Secure device-independent quantum key distribution with causally independent measurement devices. Nature Communications 2, 238 (2011).

[39] Barrett, J., Kent, A. \& Pironio, S. Maximally non-local and monogamous quantum correlations. Physical Review Letters 97, 170409 (2006).

[40] Barrett, J., Colbeck, R. \& Kent, A. Memory attacks on device-independent quantum cryptography. Physical Review Letters 110, 010503 (2013). e-print arXiv:1201.4407 (2012).

[41] R. Colbeck, Quantum And Relativistic Protocols For Secure Multi-Party Computation, University of Cambridge Ph.D. dissertation, available at arXiv:0911.3814

[42] R. Colbeck and A. Kent, Journal of Physics A: Mathematical and Theoretical 44(9), 095305 (2011).

[43] S. Pironio et al., Random numbers certified by Bells theorem, Nature 464, 1021-1024, (2010)

[44] J. Barrett, R. Colbeck, A. Kent, Unconditionally secure device-independent quantum key distribution with only two devices, Phys. Rev. A, 86 (2012)

[45] U. Vazirani, T. Vidick, Certifiable Quantum Dice - Or, testable exponential randomness expansion, arXiv:1111.6054(2011).

[46] U. Vazirani, T. Vidick, Fully Device-Independent Quantum Key Distribution, Phys. Rev. Lett., 14, (2014)

[47] R. Colbeck, R. Renner, Free randomness can be amplified, Nature Physics, 8, 450-454, (2012). 